\title{
Supplementary information for: Osmolyte Induced Changes in Peptide Conformational Ensemble Correlate with Slower Amyloid Aggregation: a Coarse-Grained Simulation Study
}

Shahar Sukenik $^{\mathrm{a}, \mathrm{b}}$, Liel Sapir ${ }^{\mathrm{a}}$, and Daniel Harries ${ }^{\mathrm{a}}$

anstitute of Chemistry and the Fritz Haber Research Center, The Hebrew University, Jerusalem, Israel; ${ }^{b}$ Current address: Department of Chemistry, University of Illinois, Urbana, IL 61801, USA.

\section{S1. Simulations}

Table S1 - All coarse grained simulations conducted in this study and corresponding composition

\begin{tabular}{|l|l|l|l|l|l|}
\hline & peptide $^{\mathrm{a}}$ & Water $^{\mathrm{a}}$ & Sorbitol $^{\mathrm{a}}$ & Time $(\mu \mathrm{s})$ & Runs \\
\hline Water & 1 & 2252 & 0 & 2 & 5 \\
\hline $1.5 \mathrm{~m}$ sorbitol & 1 & 2311 & 250 & 2 & 5 \\
\hline $1.1 \mathrm{~m}$ sorbitol & 1 & 2461 & 200 & 2 & 5 \\
\hline $0.8 \mathrm{~m}$ sorbitol & 1 & 2611 & 150 & 2 & 5 \\
\hline $0.65 \mathrm{~m}$ sorbitol & 1 & 2686 & 125 & 2 & 5 \\
\hline $0.4 \mathrm{~m}$ sorbitol & 1 & 2812 & 83 & 2 & 5 \\
\hline Water large & 216 & 486432 & 0 & 1 & 4 \\
\hline Sorbitol large & 216 & 499176 & 54000 & 1 & 3 \\
\hline $0.1 \mathrm{~m}$ sorbitol & 0 & 36000 & 256 & 1 & 1 \\
\hline $0.2 \mathrm{~m}$ sorbitol & 0 & 28800 & 416 & 1 & 1 \\
\hline $0.3 \mathrm{~m}$ sorbitol & 0 & 28800 & 624 & 1 & 1 \\
\hline $0.4 \mathrm{~m}$ sorbitol & 0 & 28800 & 832 & 1 & 1 \\
\hline $0.5 \mathrm{~m}$ sorbitol & 0 & 17776 & 640 & 1 & 1 \\
\hline $0.6 \mathrm{~m}$ sorbitol & 0 & 28800 & 1248 & 1 & 1 \\
\hline $0.7 \mathrm{~m}$ sorbitol & 0 & 28800 & 1448 & 1 & 1 \\
\hline $0.8 \mathrm{~m}$ sorbitol & 0 & 28800 & 1656 & 1 & 1 \\
\hline $0.9 \mathrm{~m}$ sorbitol & 0 & 28800 & 1864 & 1 & 1 \\
\hline $1.0 \mathrm{~m}$ sorbitol & 0 & 14400 & 1040 & 1 & 1 \\
\hline $1.1 \mathrm{~m}$ sorbitol & 0 & 28800 & 2280 & 1 & 1 \\
\hline $1.2 \mathrm{~m}$ sorbitol & 0 & 28800 & 2488 & 1 & 1 \\
\hline $1.3 \mathrm{~m}$ sorbitol & 0 & 28800 & 2696 & 1 & 1 \\
\hline $1.4 \mathrm{~m}$ sorbitol & 0 & 28800 & 2904 & 1 & 1 \\
\hline $1.5 \mathrm{~m}$ sorbitol & 0 & 13328 & 1440 & 1 & 1 \\
\hline a number $\mathrm{p}$ partic & & & & \\
\hline
\end{tabular}

${ }^{\mathrm{a}}$ number of particles in simulations 


\section{Simulation details}

All simulations were performed with the GROMACS package. ${ }^{1-4}$ The Replica Exchange Molecular Dynamics (REMD) simulation ${ }^{5}$ of the peptide in pure water was conducted using the AMBER ff99sb-ildn-NMR force field ${ }^{6-9}$ and the TIP4Pew water model. ${ }^{10}$ All bond lengths to hydrogen atoms were kept constant with the LINCS algorithm. ${ }^{11}$ The simulation consisted of 44 replicas spanning the temperature range $277-465 \mathrm{~K}$, where temperatures were assigned so as to achieve an acceptance rate of $0.2 .^{12}$ The dynamics were carried with periodic boundary conditions using a time-step of $2 \mathrm{fs}$, and replica exchange attempts every 1 ps. Temperatures were held constant using the Nosé-Hoover thermostat with a 1 ps coupling constant. ${ }^{13,14}$ Electrostatic calculations were performed using the particle-mesh Ewald (PME) method with $1 \AA$ grid spacing. ${ }^{15,16}$ Van der Waals interactions were truncated smoothly with a switching distance of $10 \AA$ and a cutoff distance of $12 \AA$, and by also accounting for long-range dispersion corrections. The REMD simulation was performed in the NVT ensemble using the volume calibrated by an isobaric-isothermic ensemble simulations at ambient temperature, $\mathrm{T}=298 \mathrm{~K}$, and pressure of $\mathrm{P}=1 \mathrm{~atm}$. Pressure in this NPT production was kept constant using the Parrinello-Rahman barostat with a 1 ps coupling constant and compressibility of $4.5 \cdot 10^{-5}$ $\operatorname{bar}^{-1} \cdot{ }^{17,18}$ Of the $500 \mathrm{~ns}$ collected in the REMD production (per replica), only the last 300 ns were used for analysis.

For the molecular dynamics simulations of aqueous sorbitol, we used the TIP4Pew water model and the GLYCAM06 force-field. ${ }^{19}$ The partial charges of sorbitol (Figure S1) were reassigned following the standard procedure ${ }^{20-22}$ by using the Gaussian 03 package $^{23}$, R.E.D tools, ${ }^{24,25}$ and Resp program. ${ }^{26}$ The derived partial charges are listed in Table S1. The sorbitol simulations were run in the isobaric-isothermic ensemble, as mentioned above. All other simulation details are as detailed above. For the charge calculations we used snapshots of the latter half of a $200 \mathrm{~ns}$ simulation.

Coarse-grained simulations were run using MARTINI forcefield v2.1 $1^{27,28}$, with a timestep of $0.04 \mathrm{ps}$ for binary solutions and of $0.01 \mathrm{ps}$ for peptide simulation. The relatively short timestep for peptide simulations is used to allow stable dihedral angles calculations ${ }^{29}$. Simulation settings were set according to recommended MARTINI parameters. Non-bonded parameters were used with a cutoff of $1.2 \mathrm{~nm}$ and a shifted function from 0.9 to $1.2 \mathrm{~nm}$. Temperature was controlled using the $\mathrm{V}$-rescale algorithm, with a time constant of 1 ps. Pressure was controlled using a Parinello-Rachman barostat with a time constant of $12 \mathrm{ps}$, compressibility of $3 \cdot 10^{-4} \mathrm{bar}^{-1}$, and a reference pressure of 1 bar for all simulation boxes. 


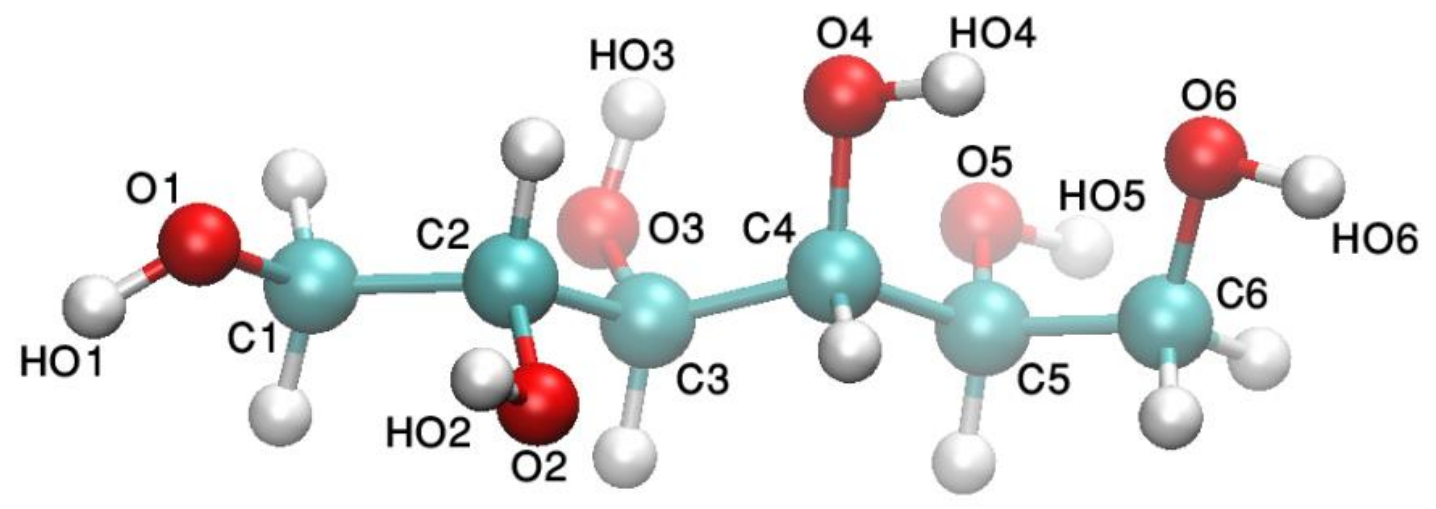

Fig. S1. A sorbitol molecule in the CPK representation.

Table S2. The derived partial charges of sorbitol in the GLYCAM force field. Atom names are as in Figure S1.

\begin{tabular}{|c|c|}
\hline Atoms & Partial charge \\
\hline C1, C6 & 0.273 \\
\hline O1, O6 & -0.696 \\
\hline HO1,HO6 & 0.423 \\
\hline C2,C3,C4,C5 & 0.288 \\
\hline O2,O3,O4,O5 & -0.714 \\
\hline HO2,HO3,HO4,HO5 & 0.426 \\
\hline All aliphatic hydrogens & 0.0 \\
\hline
\end{tabular}




\section{S2. REMD simulations analysis}
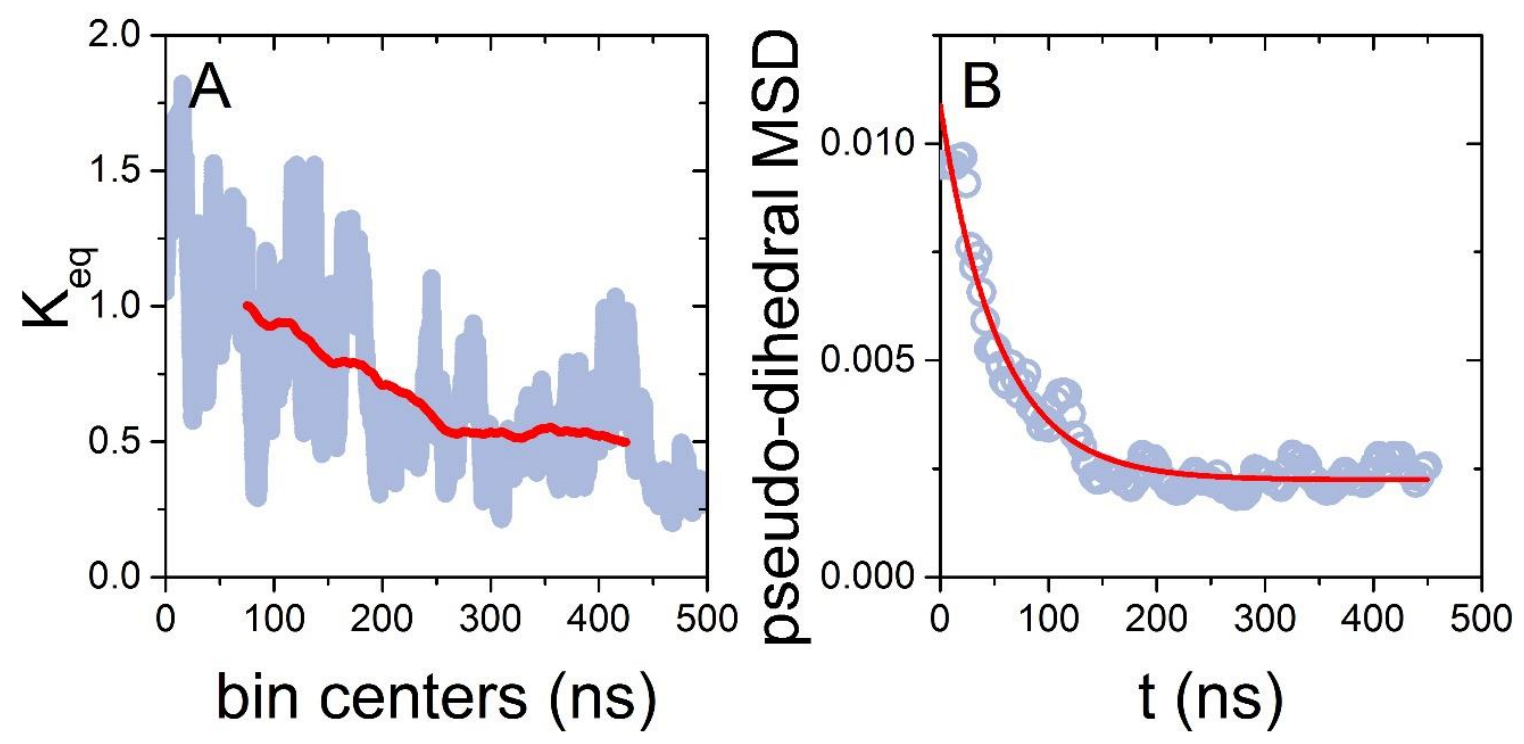

Fig. S2. Convergence of all-atom REMD simulations. (A) Convergence of folding equilibrium constant $\mathrm{K}_{\mathrm{eq}}$ from the replica at $\mathrm{T}=298 \mathrm{~K}$. $\mathrm{K}_{\mathrm{eq}}$ was calculated using the RMSD with respect to a representative folded conformation at each frame, and the cutoff for the folded ensemble was set to $\mathrm{RMSD} \leq 2.0 \AA$. The plot shows the running average of $\mathrm{K}_{\mathrm{eq}}$, where each point represents the center of an averaging window of $150 \mathrm{~ns}$, spanning $75 \mathrm{~ns}$ to the left and $75 \mathrm{~ns}$ to the right of each point (shown in red). This running average is overlaid on the averaged data of non-overlapping, $5 \mathrm{~ns}$ windows (grey). The average taken from the 175-325 ns window (centered at $250 \mathrm{~ns}$ ) already shows the same $\mathrm{K}_{\mathrm{eq}}$ as $425 \mathrm{~ns}$ (within statistical error), demonstrating the equilibrium constant is well converged. (B) Sum of mean-square-differences of all pseudo-dihedral angle distributions between a $50 \mathrm{~ns}$ running time window and the final selected dihedral distributions shown in Fig. $\mathrm{S} 3$. The red line is an exponential fit to the data in grey. Final pseudo-dihedral angles are taken from the average of the last $300 \mathrm{~ns}$ of this REMD replica where both peptide equilibrium (panel A) and dihedral angles (panel B) are well converged. 

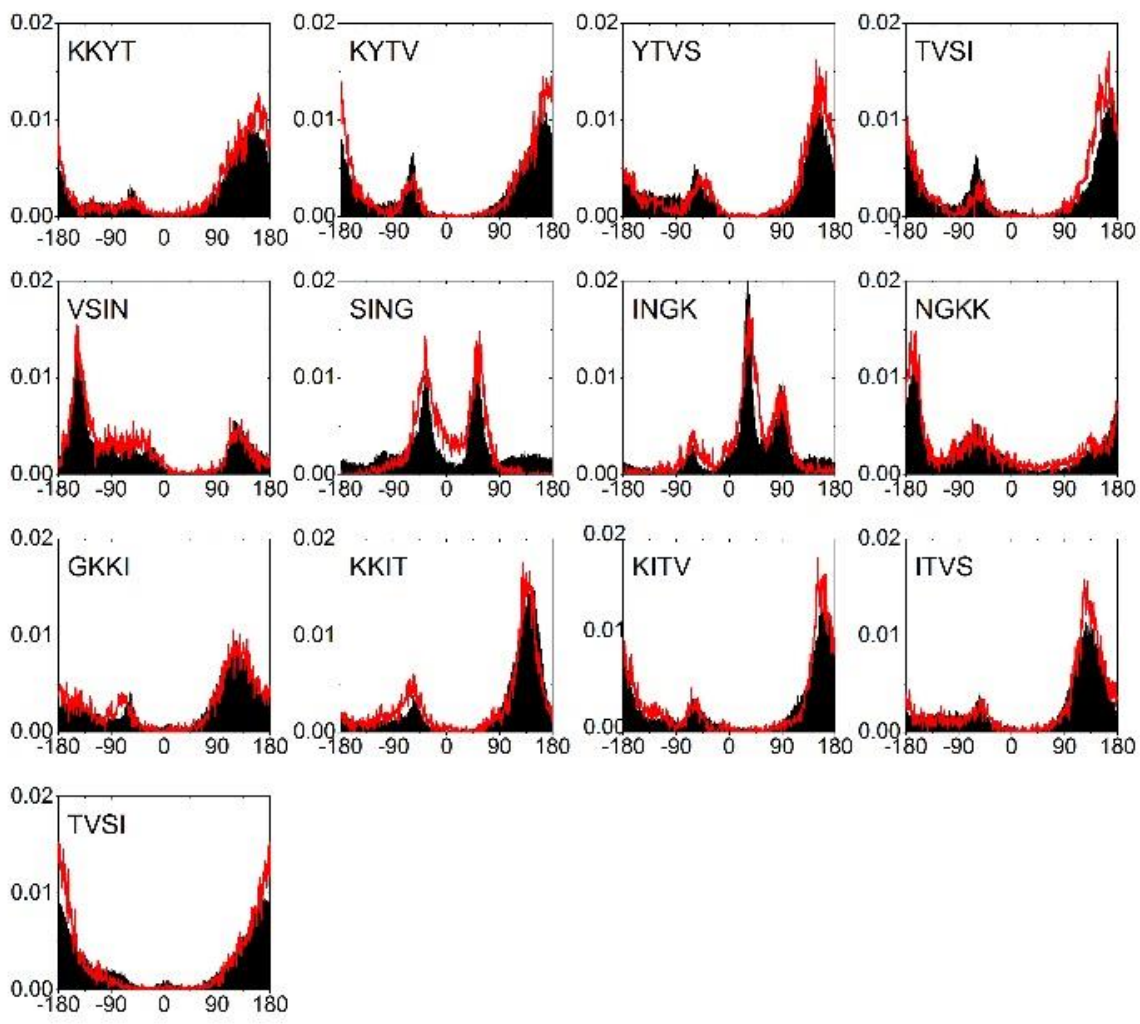

Fig. S3. Resulting pseudo-dihedral angle probability distribution in CG peptide calibrated simulations (red lines) overlaid on distributions from all-atom REMD (black bars) shows good agreement between both models.

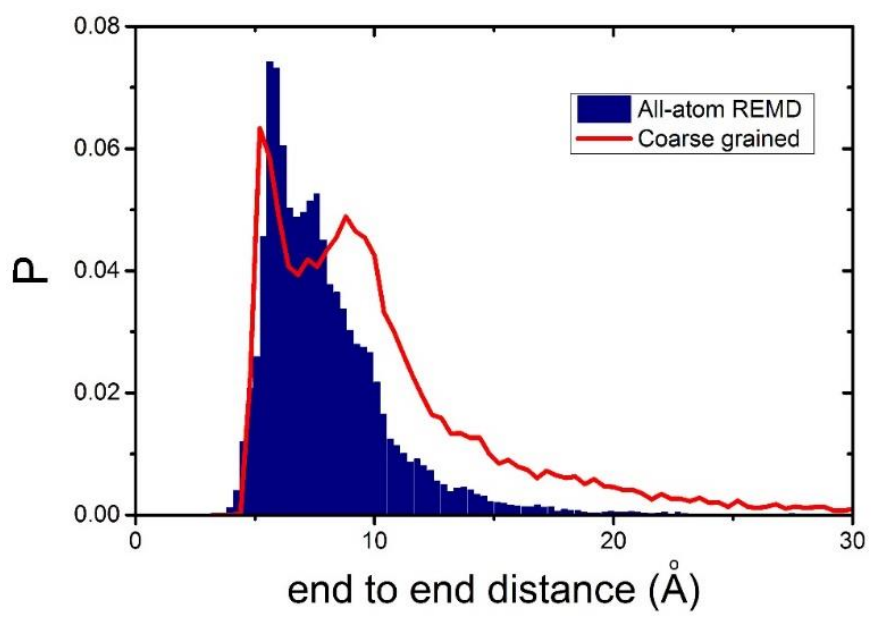

Fig. S4. Comparison of end-to-end distance between CG and all-atom model of met16. 


\section{S3. Osmotic pressure of sorbitol model}

In order to evaluate how well the sorbitol model reproduces experimentally known solution properties, we calculated the osmotic pressure of the sorbitol model in binary solutions with MARTINI waters using two different methods. The first method uses the sorbitol-sorbitol radial distribution function, $g_{s s}(r)$ to calculate the second virial coefficient $B$, through the relation ${ }^{30}$

$$
B=-2 \pi N_{a v} \int_{r=0}^{\infty}\left(g_{s s}(r)-1\right) r^{2} d r
$$

with $r$ the distance from the center sorbitol's center of mass, and $N_{a v}$ Avogadro's constant. Once determined, $B$ can be used to calculate the osmotic pressure $\Pi$ at low sorbitol concentrations using sorbitol molality $m_{S}$, and the mass to volume density of the solution, $\boldsymbol{d}$ :

$$
\Pi \cong m_{s}+B d m_{s}^{2}
$$

A second method to obtain the osmotic pressure is through the use of the Kirkwood-Buff theory of solutions, as we describe in Ref. 31. Briefly, the derivative of the osmotic pressure with respect to water density is related to the Kirkwood-Buff integrals $\mathcal{G}_{s w}^{\infty}$ and $\mathcal{G}_{w w}^{\infty}$ (the former integral, for example, is shown for $1 \mathrm{~mol} / \mathrm{kg}$ Fig. 2D, and discussed in the main text) by:

$$
\left(\frac{\partial \Pi}{\partial \rho_{w}}\right)=\frac{-m_{w}}{\rho_{w}\left(1+\rho_{w} \mathcal{G}_{w w}^{\infty}-\rho_{w} \mathcal{G}_{s w}^{\infty}\right)} .
$$

Here, $\rho_{w}$ is the water-bead number density, and $m_{w}$ is the pure water-bead molality. Integrating over a second order polynomial fit to the resulting values for the derivative with respect to $\rho_{w}$, and setting the integration constant so that $\Pi=0$ in pure water, yields the simulated osmotic pressure. While this method gives the exact osmotic pressure, it is numerically noisier than the first estimate as two KBIs are involved rather than one.

Fig S5 shows the results from both methods, and compares them with experimental (red line) and ideal (van 't Hoff, black line) osmotic pressures. Both methods used to calculate the pressure from simulations carry errors with them. These are mainly associated with radial distributions integrals, which are notoriously difficult to converge. Despite this, a qualitatively good fit, showing a slight yet positive deviation from ideal osmotic behavior is apparent, in agreement with experiments. The deviation from experimental results increases, however, as sorbitol concentrations increase. At the concentration used in our peptide aggregation study ( $1 \mathrm{~mol} / \mathrm{kg}$ ) the osmotic pressure shows a close agreement with experiment. Important to the coarse-grained aspect of our simulations, all calculations performed here do not utilize any special or arbitrary fitting factors to account for the 4-to-1 mapping native to MARTINI. We conclude that despite 
the known drawbacks of the coarse grained representation, our model manages to reproduce experimental solution thermodynamic parameters to good accuracy.

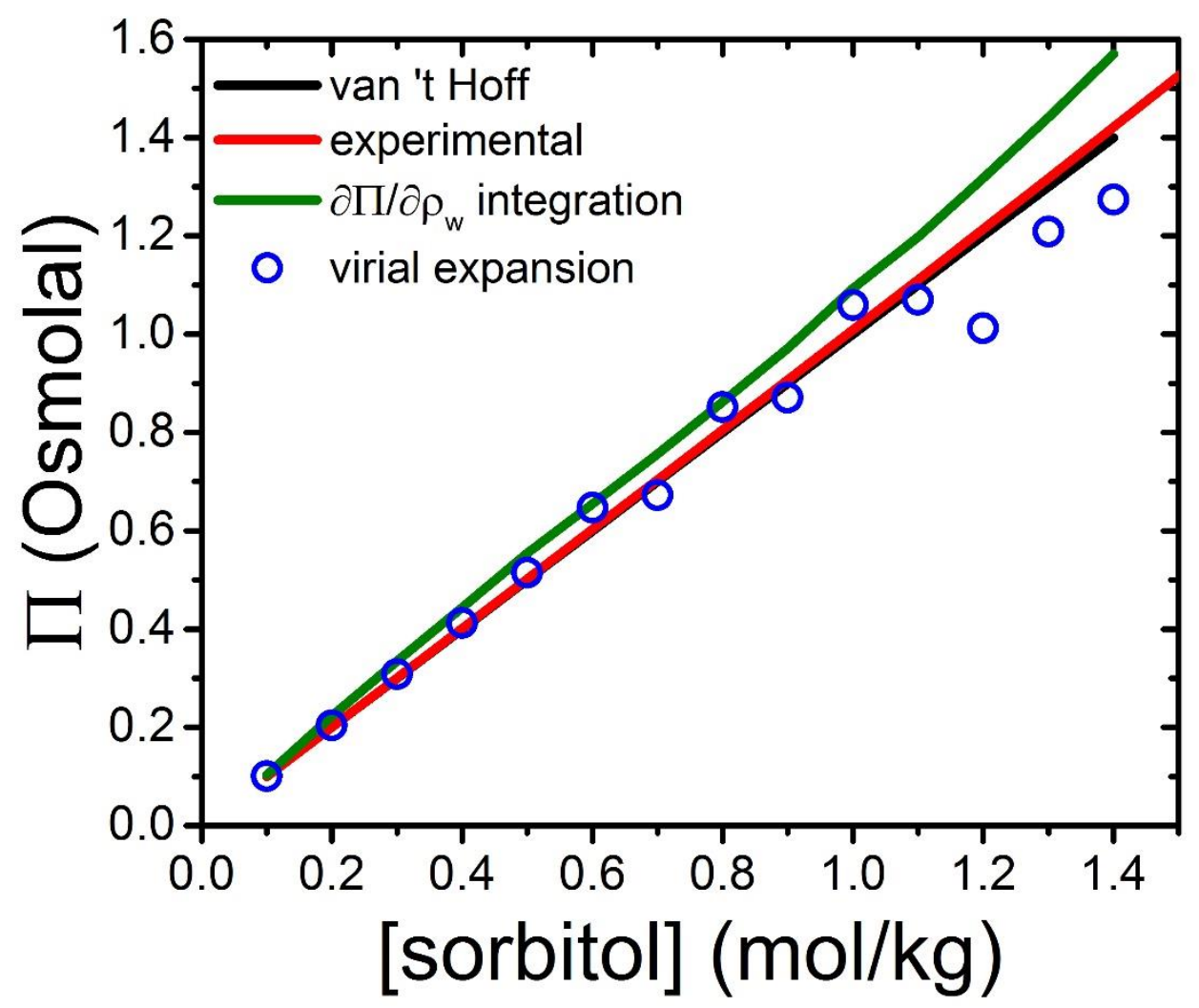

Fig. S5. Comparison between simulated and experimental osmotic pressures. Two methods of calculation for the osmotic pressure, discussed above, are shown as blue symbols and green line, and compared with experimental data shown as the red line. Small positive deviations from ideal osmotic pressure (in black) are observed for both simulated and experimental data at the relevant concentrations used in this study. 


\section{S4. Preferential interaction of sorbitol with the peptide}

An additional method to obtain the effect of cosolute on protein folding free-energy is through the direct measurement of the number of sorbitol excluding waters, where

$$
\Delta \Gamma=\left[N_{W}\left(1-\frac{N_{S} / N_{W}}{n_{S} / n_{W}}\right)\right]_{\text {folded }}-\left[N_{W}\left(1-\frac{N_{S} / N_{W}}{n_{S} / n_{W}}\right)\right]_{\text {unfolded }}
$$

Here, $N$ relates to the number of molecules in the vicinal area of the peptide, while $n$ is the number of molecules in the bulk area, far from the peptide, and the subscripts $S$ and $W$ represent cosolute and water models respectively. A cutoff between the vicinal and bulk regions is determined by the region of $\mathrm{g}(\mathrm{r})$ convergence (Fig. 2D). The number for $\Delta \Gamma$ is obtained by averaging the limiting value of the curve shown in Fig. S6 at the region where no more changes (apart from the undulations known to occur in coarse-grained liquids ${ }^{32,33}$, typically 15-20 $\AA$ from the peptide surface). The difference $\Delta \Gamma$ can then be related to the $m$-value (as described in the main text) through the relation

$$
m=\frac{R T}{13.9} \Delta \Gamma
$$

where 13.9 is the number (in moles) of water beads in $1 \mathrm{~kg}$ of pure MARTINI water, $R$ is the universal gas constant, and $T$ is the temperature.

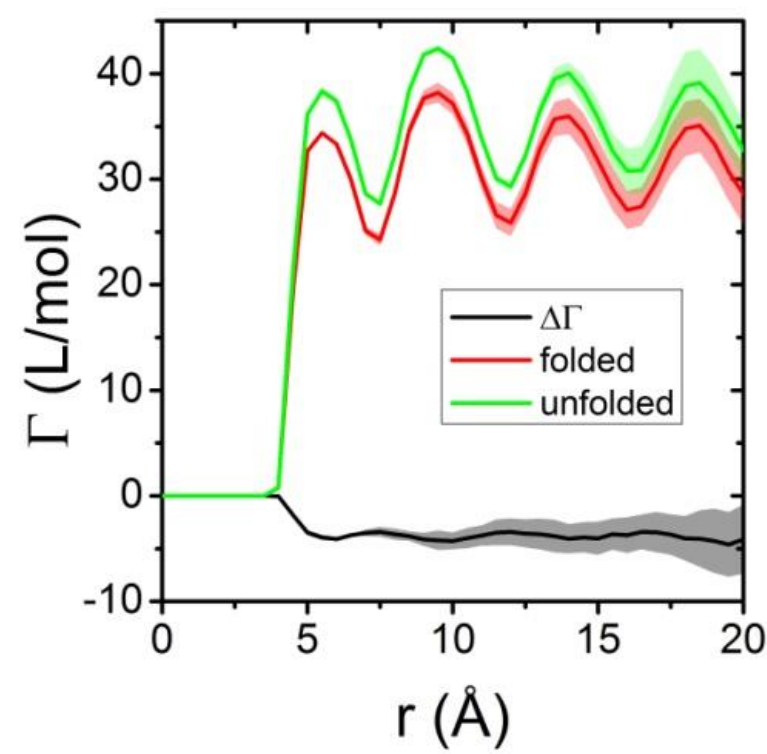

Fig S6. Change in preferential interaction of water with peptide shows difference between the folded and unfolded ensemble in the presence of $1 \mathrm{~mol} / \mathrm{kg}$ sorbitol. The value of $\Delta \Gamma$ is proportional to the $m$-value discussed in the text. The overall free energy change upon folding in these conditions is $m=-0.9 \pm 0.2 \mathrm{~kJ} / \mathrm{mol}$. 


\section{S5. SASA changes upon folding of CG peptide}
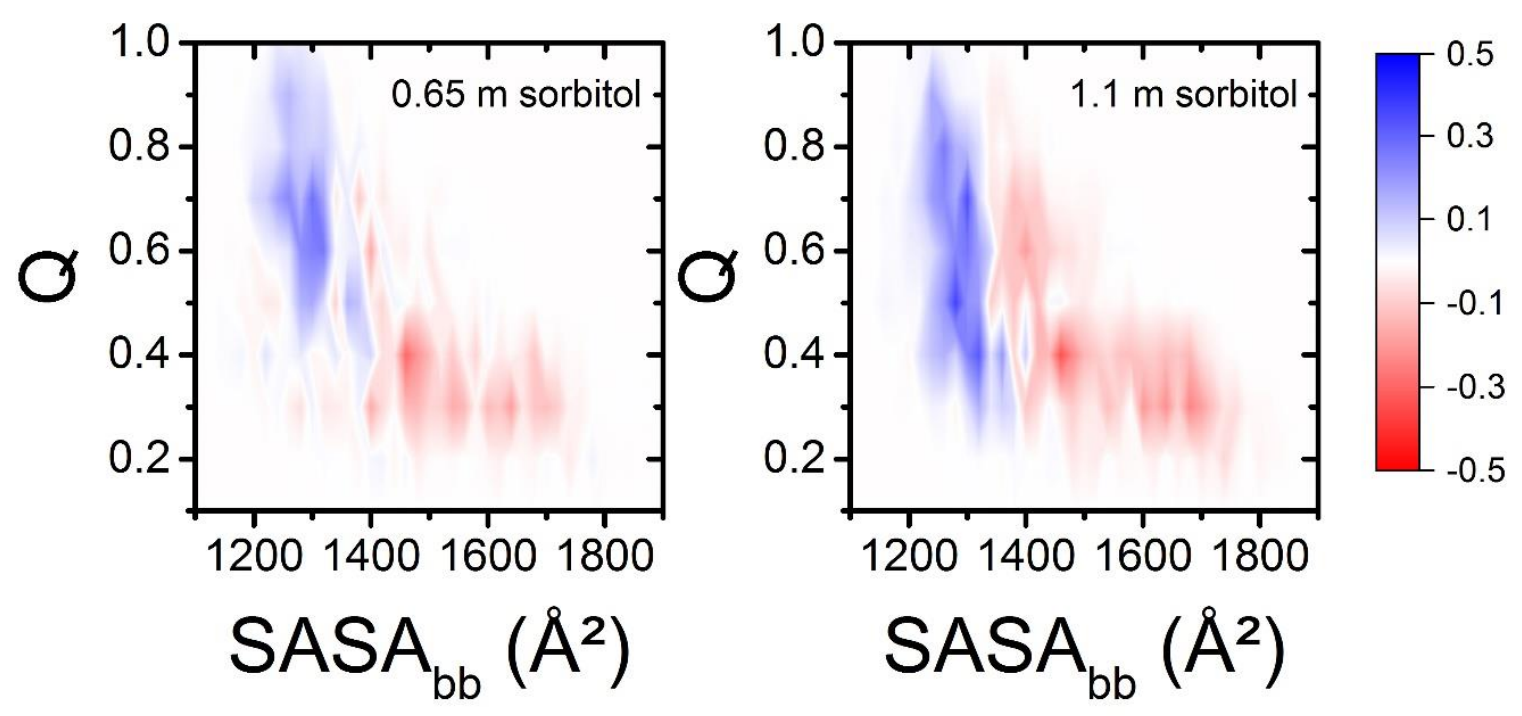

Fig S7. Changes in Q vs. backbone SASA distributions due to the presence of sorbitol. Values are calculated by subtracting the probability distributions in the presence of sorbitol from those in the absence of sorbitol. Red regions show a decrease in probability, while blue regions show an increase, and color intensity indicates the magnitude of distribution change (in percent). The figures clearly show that the presence of sorbitol differentiates high vs. low SASA conformations, but does not have a similarly clear cutoff for Q.

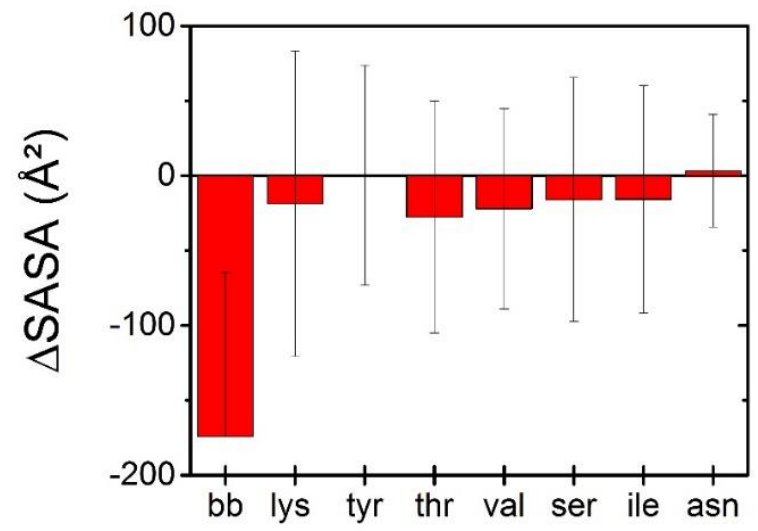

Fig S8. Changes in SASA upon folding pf CG peptide in water for backbone and sidechains of different amino acids. For each ensemble defined by the SASA cutoff specified in the text, an average SASA is obtained using the VMD measure algorithm. The probe size is set to 2.35, the size of the MARTINI water bead. The difference upon folding is shown. Error bars are the propagated standard deviations of the ensemble averages. 


\section{S6. Kinetic model for amyloid growth}

The master equation used here to numerically fit the experimental result is based on a master equation originally developed by Knowles et al. ${ }^{34}$ to model amyloid aggregation kinetics. We previously modified this scheme to include monomer detachment from formed fibrils. This addition was necessary to explain our experimental observables for met16 aggregation in the presence of various osmolytes and polymers, including sorbitol. ${ }^{35}$ Here we add yet another term, the attachment of formed fibrils to each other. This is necessary because our simulated trajectories show that a major part of elongation results from attachment of formed fibrils to each other. The final form of the kinetic scheme is formulated as a discrete master equation for clusters of size $i$

$$
\begin{aligned}
& \frac{d P_{i}}{d t}=k_{o n}\left[P_{1}\left(P_{i-1}-P_{i}\right)\right]+k_{o f f}\left[P_{i+1}-P_{i}\right]+k_{b}\left[\sum_{j=i+2}^{N} P_{j}+P_{2 i}-(i-3) P_{i}\right]+ \\
& +k_{a}\left[\sum_{k+j=i} P_{j} P_{k}-P_{i}\left(\sum_{j=2}^{N} P_{j}\right)-P_{i}^{2}\right]
\end{aligned}
$$

Here $P_{i}$ are probabilities of monomers to exist in a cluster of size $i, k_{\text {on }}$ and $k_{\text {off }}$ are rates of monomer attachment and detachment to formed fibrils, and $k_{b}$ and $k_{a}$ are rates of fibril breakage and attachment to other fibrils, respectively. An additional term of the form $k_{n} P_{1}^{2}$ describes nucleation from two monomers, where the nucleus is of size $i=2$, and exists for monomers and dimers only. The rate of fibril-to-fibril attachment, $k_{a}$ was necessary to fit our simulations results but was not necessary to fit the aggregation data of our experimental work. This is because the ThT fluorescence assay used as a probe in our experiments cannot discern events of fibril attachment. 


\section{References:}

(1) Hess, B.; Kutzner, C.; Van Der Spoel, D.; Lindahl, E. GRGMACS 4: Algorithms for Highly Efficient, Load-Balanced, and Scalable Molecular Simulation. J. Chem. Theory Comput. 2008, 4 (3), 435-447.

(2) Van Der Spoel, D.; Lindahl, E.; Hess, B.; Groenhof, G.; Mark, A. E.; Berendsen, H. J. C. GROMACS: Fast, Flexible, and Free. J. Comput. Chem. 2005, 26 (16), 1701-1718.

(3) Juréus, A.; Langel, Ü. Galanin and Galanin Antagonists. Acta Chim. Slov. 1996, 43 (1), $51-60$.

(4) Berendsen, H. J. C.; van der Spoel, D.; van Drunen, R. GROMACS: A Message-Passing Parallel Molecular Dynamics Implementation. Computer Physics Communications. September 1995, pp 43-56.

(5) Sugita, Y.; Okamoto, Y. Replica-Exchange Molecular Dynamics Method for Protein Folding. Chem. Phys. Lett. 1999, 314 (1-2), 141-151.

(6) Hornak, V.; Abel, R.; Okur, A.; Strockbine, B.; Roitberg, A.; Simmerling, C. Comparison of Multiple Amber Force Fields and Development of Improved Protein Backbone Parameters. Proteins Struct. Funct. Bioinforma. 2006, 65 (3), 712-725.

(7) Lindorff-Larsen, K.; Piana, S.; Palmo, K.; Maragakis, P.; Klepeis, J. L.; Dror, R. O.; Shaw, D. E. Improved Side-Chain Torsion Potentials for the Amber ff99SB Protein Force Field. Proteins Struct. Funct. Bioinforma. 2010, 78 (8), 1950-1958.

(8) Li, D.-W.; Brüschweiler, R. NMR-Based Protein Potentials. Angew. Chemie 2010, 122 (38), 6930-6932.

(9) Beauchamp, K. A.; Lin, Y.-S.; Das, R.; Pande, V. S. Are Protein Force Fields Getting Better? A Systematic Benchmark on 524 Diverse NMR Measurements. J. Chem. Theory Comput. 2012, 8 (4), 1409-1414.

(10) Horn, H. W.; Swope, W. C.; Pitera, J. W.; Madura, J. D.; Dick, T. J.; Hura, G. L.; HeadGordon, T. Development of an Improved Four-Site Water Model for Biomolecular Simulations: TIP4P-Ew. J. Chem. Phys. 2004, 120 (20), 9665-9678.

(11) Hess, B.; Bekker, H.; Berendsen, H. J. C.; Fraaije, J. G. E. M. LINCS: A Linear Constraint Solver for Molecular Simulations. J. Comput. Chem. 1997, 18 (12), 1463-1472.

(12) Garcia, A. E.; Herce, H.; Paschek, D. Simulations of Temperature and Pressure Unfolding of Peptides and Proteins with Replica Exchange Molecular Dynamics. In Annual Reports in Computational Chemistry; Chemistry, D. C. S. B. T.-A. R. in C., Ed.; Elsevier, 2006; Vol. Volume 2, pp 83-95.

(13) Nosé, S. A Molecular Dynamics Method for Simulations in the Canonical Ensemble. Mol. Phys. 1984, 52 (2), 255-268. 
(14) Hoover, W. G. Canonical Dynamics: Equilibrium Phase-Space Distributions. Phys. Rev. A 1985, 31 (3), 1695-1697.

(15) Darden, T.; York, D.; Pedersen, L. Particle Mesh Ewald: An N $\log (\mathrm{N})$ Method for Ewald Sums in Large Systems. J. Chem. Phys. 1993, 98 (12), 10089.

(16) Essmann, U.; Perera, L.; Berkowitz, M. L.; Darden, T.; Lee, H.; Pedersen, L. G. A Smooth Particle Mesh Ewald Method. J Chem Phys 1995, 103 (November), 8577-8593.

(17) Parrinello, M. Polymorphic Transitions in Single Crystals: A New Molecular Dynamics Method. J. Appl. Phys. 1981, 52 (12), 7182.

(18) Nosé, S.; Klein, M. L. Constant Pressure Molecular Dynamics for Molecular Systems. Mol. Phys. 1983, 50 (5), 1055-1076.

(19) Kirschner, K. N.; Yongye, A. B.; Tschampel, S. M.; González-Outeiriño, J.; Daniels, C. R.; Foley, B. L.; Woods, R. J. GLYCAM06: A Generalizable Biomolecular Force Field. Carbohydrates. J. Comput. Chem. 2008, 29 (4), 622-655.

(20) Basma, M.; Sundara, S.; Calgan, D.; Vernali, T.; Woods, R. J. Solvated Ensemble Averaging in the Calculation of Partial Atomic Charges. J. Comput. Chem. 2001, 22 (11), $1125-1137$.

(21) Francl, M. M.; Carey, C.; Gange, D. M. Charges Fit to Electrostatic Potentials . 11 . Can Atomic Charges Be Unambiguously Fit to Electrostatic Potentials? J. Comp. Chem. 1995, 17 (3), 367-383.

(22) Yongye, A. B.; Foley, B. L.; Woods, R. J. On Achieving Experimental Accuracy from Molecular Dynamics Simulations of Flexible Molecules: Aqueous Glycerol. J. Phys. Chem. A 2008, 112 (12), 2634-2639.

(23) Frisch, M. J.; Trucks, G. W.; Schlegel, H. B.; Scuseria, G. E.; Robb, M. A.; Cheeseman, J. R.; Montgomery, J. A.; Vreven, J. T.; Kudin, K. N.; Burant, J. C.; Millam, J. M.; Iyengar, S. S.; Tomasi, J.; Barone, V.; Mennucci, B.; Cossi, M.; Scalmani, G.; Rega, N.; Petersson, G. A.; Nakatsuji, H.; Hada, M.; Ehara, M.; Toyota, K.; Fukuda, R.; Hasegawa, J.; Ishida, M.; Nakajima, T.; Honda, Y.; Kitao, O.; Nakai, H.; Klene, M.; Li, X.; Hratchian, J. E. K. H. P.; Cross, J. B.; Adamo, C.; Jaramillo, J.; Gomperts, R.; Stratmann, R. E.; Yazyev, O.; Austin, A. J.; Cammi, R.; Pomelli, C.; Ochterski, J.; Ayala, P. Y.; Morokuma, K.; Voth, G. A.; Salvador, P.; Dannenberg, J. J.; Zakrzewski, V. G.; Dapprich, S.; Daniels, A. D.; Strain, M. C.; Farkas, O.; Malick, D. K.; Rabuck, A. D.; Raghavachari, K.; Foresman, J. B.; Ortiz, J. V.; Cui, Q.; Baboul, A. G.; Clifford, S.; Cioslowski, J.; Stefanov, B. B.; Liu, G.; Liashenko, A.; Piskorz, P.; Komaromi, I.; Martin, R. L.; Fox, D. J.; Keith, T.; AlLaham, M. A.; Peng, C. Y.; Nanayakkara, A.; Challacombe, M.; Gill, P. M. W.; Johnson, B.; Chen, W.; Wong, M. W.; Gonzalez, C.; Pople, J. A. Gaussian 09, Revision B.01. Guassian Inc. Gaussian, Inc.: Wallingford CT 2010.

(24) Vanquelef, E.; Simon, S.; Marquant, G.; Garcia, E.; Klimerak, G.; Delepine, J. C.; Cieplak, P.; Dupradeau, F.-Y. R.E.D. Server: A Web Service for Deriving RESP and ESP Charges and Building Force Field Libraries for New Molecules and Molecular Fragments. Nucleic Acids Res. 2011, 39 (suppl), W511-W517. 
(25) Dupradeau, F.-Y.; Pigache, A.; Zaffran, T.; Savineau, C.; Lelong, R.; Grivel, N.; Lelong, D.; Rosanski, W.; Cieplak, P. The R.E.D. Tools: Advances in RESP and ESP Charge Derivation and Force Field Library Building. Phys. Chem. Chem. Phys. 2010, 12 (28), 7821-7839.

(26) Bayly, C. I.; Bayly, C. I.; Cieplak, P.; Cieplak, P.; Cornell, W. D.; Cornell, W. D.; Kollman, P. A.; Kollman, P. A. A Well-Behaved Electrostatic Potential Based Method Using Charge Restraints for Deriving Atomic Charges-the RESP Model. J. Phys. Chem 1993, 97, 10269-10280.

(27) Marrink, S.-J.; Risselada, H. J.; Yefimov, S.; Tieleman, D. P.; de Vries, A. H. The MARTINI Force Field: Coarse Grained Model for Biomolecular Simulations. J. Phys. Chem. B 2007, 111 (27), 7812-7824.

(28) Monticelli, L.; Kandasamy, S. K.; Periole, X.; Larson, R. G.; Tieleman, D. P.; Marrink, S.J. The MARTINI Coarse-Grained Force Field: Extension to Proteins. J. Chem. Theory Comput. 2008, 4 (5), 819-834.

(29) Seo, M.; Rauscher, S.; Pomès, R.; Tieleman, D. P. Improving Internal Peptide Dynamics in the Coarse-Grained MARTINI Model: Toward Large-Scale Simulations of Amyloidand Elastin-like Peptides. J. Chem. Theory Comput. 2012, 8 (5), 1774-1785.

(30) McQuarrie, D. A.. Statistical Mechanics; University Science Books, 2000.

(31) Sapir, L.; Harries, D. Linking Trehalose Self-Association with Binary Aqueous Solution Equation of State. J. Phys. Chem. B 2011, 115 (4), 624-634.

(32) Ganguly, P.; Mukherji, D.; Junghans, C.; van der Vegt, N. F. A. Kirkwood-Buff CoarseGrained Force Fields for Aqueous Solutions. J. Chem. Theory Comput. 2012, 8 (5), 18021807.

(33) Mukherji, D.; van der Vegt, N. F. A.; Kremer, K.; Delle Site, L. Kirkwood-Buff Analysis of Liquid Mixtures in an Open Boundary Simulation. J. Chem. Theory Comput. 2012, 8 (2), 375-379.

(34) Knowles, T. P. J.; Waudby, C. A.; Devlin, G. L.; Cohen, S. I. A.; Aguzzi, A.; Vendruscolo, M.; Terentjev, E. M.; Welland, M. E.; Dobson, C. M. An Analytical Solution to the Kinetics of Breakable Filament Assembly. Science 2009, 326 (5959), 1533-1537.

(35) Sukenik, S.; Harries, D. Insights into the Disparate Action of Osmolytes and Macromolecular Crowders on Amyloid Formation. Prion 2012, 6 (1), 26-31. 


\section{Appendix 1: Forcefield paramaters for met16 peptide including REMD-calibrated dihedrals in GROMACS format}

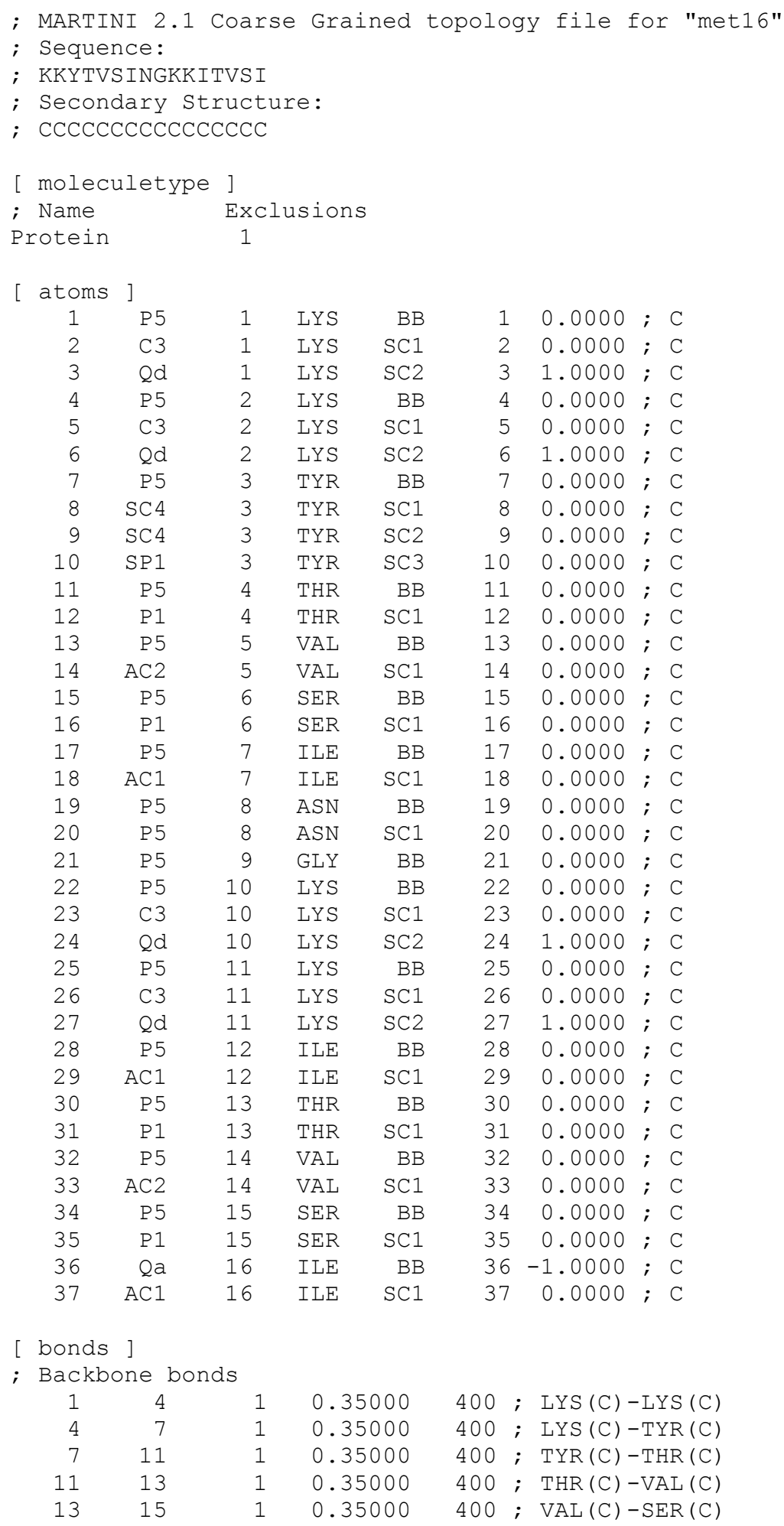




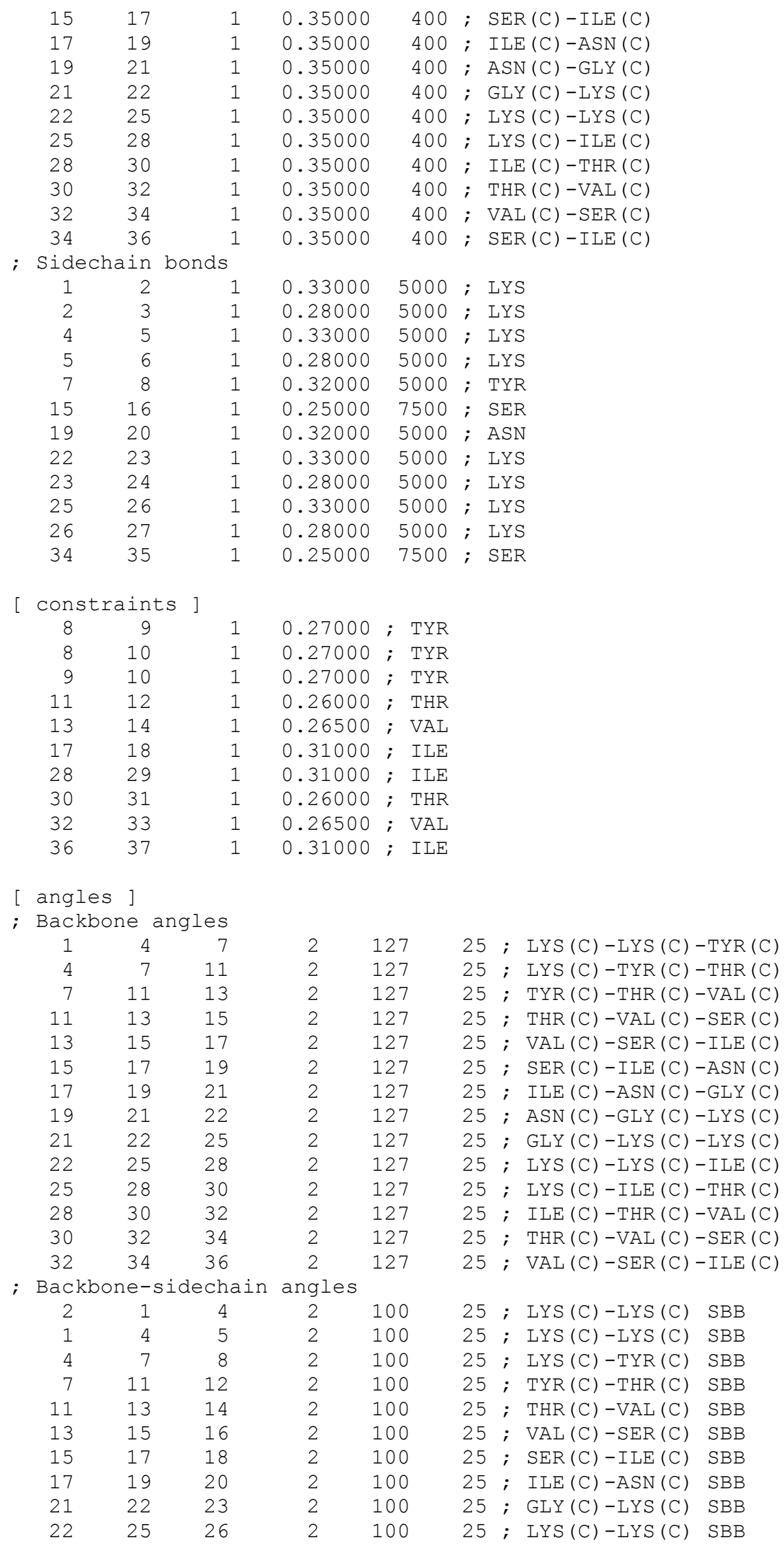




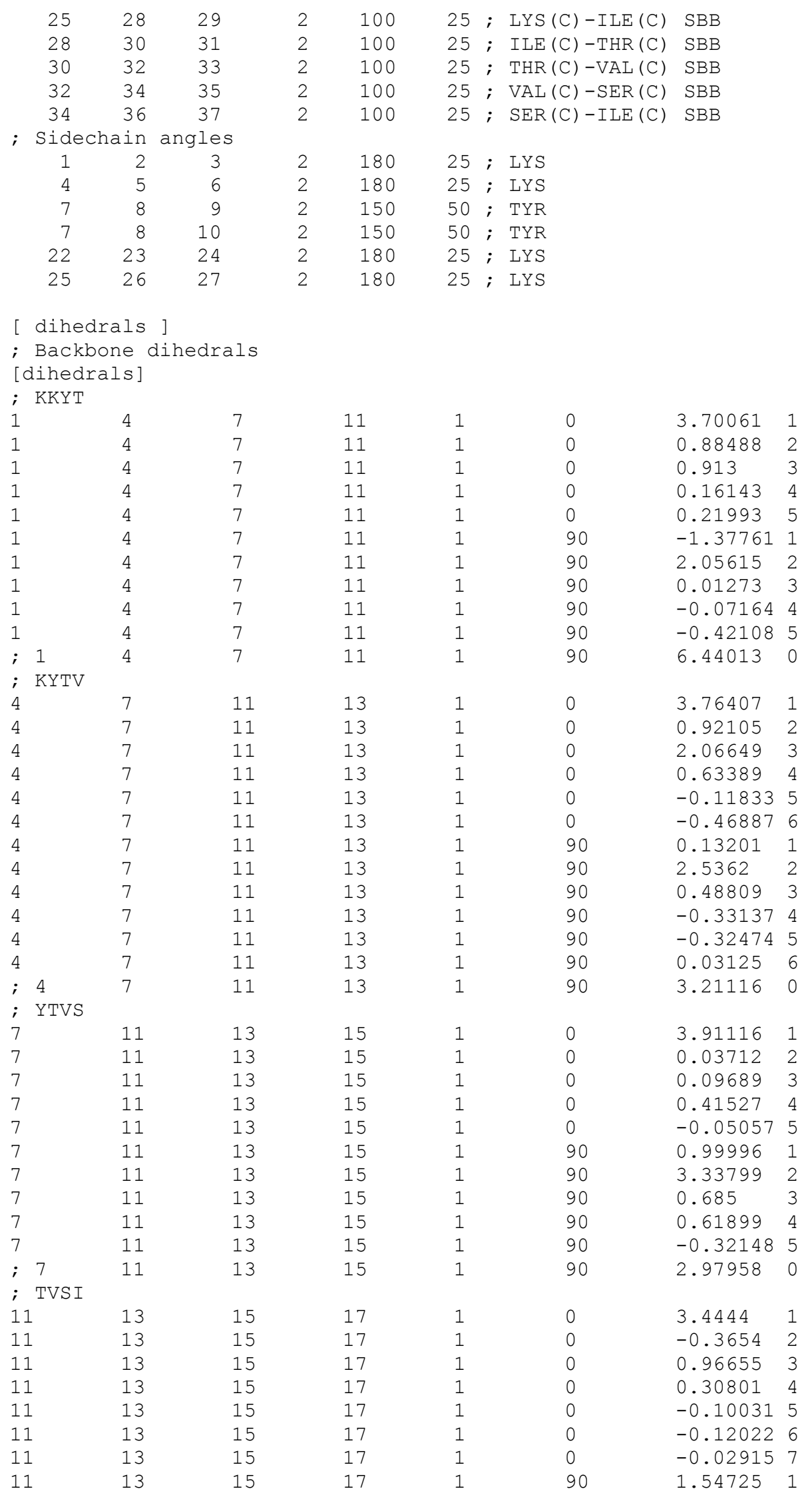




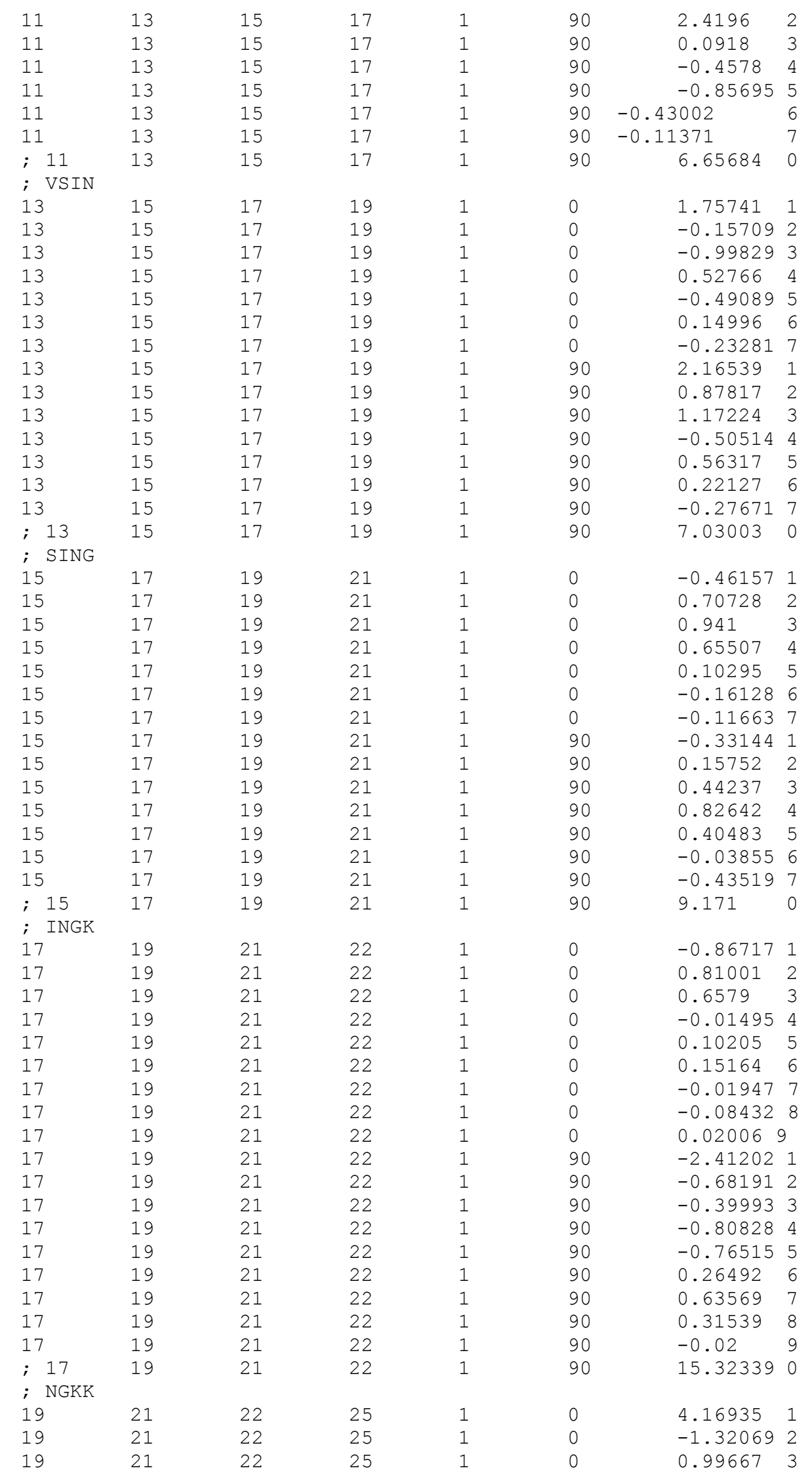




\begin{tabular}{|c|c|c|c|c|c|c|}
\hline 19 & 21 & 22 & 25 & 1 & 0 & -0.25206 \\
\hline 19 & 21 & 22 & 25 & 1 & 0 & 0.04114 \\
\hline 19 & 21 & 22 & 25 & 1 & 0 & -0.10523 \\
\hline 19 & 21 & 22 & 25 & 1 & 0 & 0.01626 \\
\hline 19 & 21 & 22 & 25 & 1 & 90 & 0.52452 \\
\hline 19 & 21 & 22 & 25 & 1 & 90 & 1.18747 \\
\hline 19 & 21 & 22 & 25 & 1 & 90 & 0.36998 \\
\hline 19 & 21 & 22 & 25 & 1 & 90 & -0.56404 \\
\hline 19 & 21 & 22 & 25 & 1 & 90 & 0.41719 \\
\hline 19 & 21 & 22 & 25 & 1 & 90 & -0.34757 \\
\hline 19 & 21 & 22 & 25 & 1 & 90 & 0.24711 \\
\hline ; 19 & 21 & 22 & 25 & 1 & 90 & 7.15402 \\
\hline ; GKKI & & & & & & \\
\hline 21 & 22 & 25 & 28 & 1 & 0 & 3.35857 \\
\hline 21 & 22 & 25 & 28 & 1 & 0 & 1.28718 \\
\hline 21 & 22 & 25 & 28 & 1 & 0 & 0.36333 \\
\hline 21 & 22 & 25 & 28 & 1 & 0 & 0.06896 \\
\hline 21 & 22 & 25 & 28 & 1 & 0 & -0.59184 \\
\hline 21 & 22 & 25 & 28 & 1 & 0 & -0.31134 \\
\hline 21 & 22 & 25 & 28 & 1 & 0 & 0.00339 \\
\hline 21 & 22 & 25 & 28 & 1 & 90 & -0.57581 \\
\hline 21 & 22 & 25 & 28 & 1 & 90 & 1.56454 \\
\hline 21 & 22 & 25 & 28 & 1 & 90 & 0.39271 \\
\hline 21 & 22 & 25 & 28 & 1 & 90 & -0.73144 \\
\hline 21 & 22 & 25 & 28 & 1 & 90 & -0.17465 \\
\hline 21 & 22 & 25 & 28 & 1 & 90 & 0.0753 \\
\hline 21 & 22 & 25 & 28 & 1 & 90 & 0.09612 \\
\hline ; 21 & 22 & 25 & 28 & 1 & 90 & 7.03307 \\
\hline ; KKIT & & & & & & \\
\hline 22 & 25 & 28 & 30 & 1 & 0 & 4.3381 \\
\hline 22 & 25 & 28 & 30 & 1 & 0 & 1.08812 \\
\hline 22 & 25 & 28 & 30 & 1 & 0 & -0.11046 \\
\hline 22 & 25 & 28 & 30 & 1 & 0 & 0.27796 \\
\hline 22 & 25 & 28 & 30 & 1 & 0 & -0.50607 \\
\hline 22 & 25 & 28 & 30 & 1 & 0 & -0.0532 \\
\hline 22 & 25 & 28 & 30 & 1 & 90 & -0.97285 \\
\hline 22 & 25 & 28 & 30 & 1 & 90 & 3.06055 \\
\hline 22 & 25 & 28 & 30 & 1 & 90 & -0.53428 \\
\hline 22 & 25 & 28 & 30 & 1 & 90 & -0.04908 \\
\hline 22 & 25 & 28 & 30 & 1 & 90 & -0.38271 \\
\hline 22 & 25 & 28 & 30 & 1 & 90 & -0.40955 \\
\hline$; 22$ & 25 & 28 & 30 & 1 & 90 & 7.23719 \\
\hline ; KITV & & & & & & \\
\hline 25 & 28 & 30 & 32 & 1 & 0 & 3.26266 \\
\hline 25 & 28 & 30 & 32 & 1 & 0 & -0.35859 \\
\hline 25 & 28 & 30 & 32 & 1 & 0 & 0.68107 \\
\hline 25 & 28 & 30 & 32 & 1 & 0 & 0.10521 \\
\hline 25 & 28 & 30 & 32 & 1 & 0 & -0.01496 \\
\hline 25 & 28 & 30 & 32 & 1 & 0 & -0.15923 \\
\hline 25 & 28 & 30 & 32 & 1 & 0 & -0.17012 \\
\hline 25 & 28 & 30 & 32 & 1 & 90 & 0.63646 \\
\hline 25 & 28 & 30 & 32 & 1 & 90 & 1.97928 \\
\hline 25 & 28 & 30 & 32 & 1 & 90 & -0.14443 \\
\hline 25 & 28 & 30 & 32 & 1 & 90 & -0.06725 \\
\hline 25 & 28 & 30 & 32 & 1 & 90 & -0.30435 \\
\hline 25 & 28 & 30 & 32 & 1 & 90 & -0.06585 \\
\hline 25 & 28 & 30 & 32 & 1 & 90 & 0.18504 \\
\hline$; 25$ & 28 & 30 & 32 & 1 & 90 & 6.94951 \\
\hline ; ITVS & & & & & & \\
\hline 28 & 30 & 32 & 34 & 1 & 0 & 3.12156 \\
\hline 28 & 30 & 32 & 34 & 1 & 0 & 1.65553 \\
\hline 28 & 30 & 32 & 34 & 1 & 0 & 0.00546 \\
\hline 28 & 30 & 32 & 34 & 1 & 0 & 0.45185 \\
\hline
\end{tabular}




\begin{tabular}{|c|c|c|c|c|c|c|}
\hline 25 & 28 & 30 & 32 & 1 & 0 & -0.04926 \\
\hline 25 & 28 & 30 & 32 & 1 & 0 & -0.23398 \\
\hline 25 & 28 & 30 & 32 & 1 & 0 & -0.2555 \\
\hline 28 & 30 & 32 & 34 & 1 & 90 & -0.17809 \\
\hline 28 & 30 & 32 & 34 & 1 & 90 & 2.6502 \\
\hline 28 & 30 & 32 & 34 & 1 & 90 & 0.25742 \\
\hline 28 & 30 & 32 & 34 & 1 & 90 & 0.00349 \\
\hline 25 & 28 & 30 & 32 & 1 & 90 & -0.26109 \\
\hline 25 & 28 & 30 & 32 & 1 & 90 & -0.12253 \\
\hline 25 & 28 & 30 & 32 & 1 & 90 & 0.12481 \\
\hline$; 28$ & 30 & 32 & 34 & 1 & 90 & 4.95073 \\
\hline ; TVSI & & & & & & \\
\hline 30 & 32 & 34 & 36 & 1 & 0 & 4.37967 \\
\hline 30 & 32 & 34 & 36 & 1 & 0 & -0.29652 \\
\hline 30 & 32 & 34 & 36 & 1 & 0 & -0.13548 \\
\hline 30 & 32 & 34 & 36 & 1 & 0 & -0.73646 \\
\hline 25 & 28 & 30 & 32 & 1 & 0 & -0.36224 \\
\hline 30 & 32 & 34 & 36 & 1 & 90 & -0.05251 \\
\hline 30 & 32 & 34 & 36 & 1 & 90 & 0.69862 \\
\hline 30 & 32 & 34 & 36 & 1 & 90 & -0.09365 \\
\hline 30 & 32 & 34 & 36 & 1 & 90 & -0.23855 \\
\hline 25 & 28 & 30 & 32 & 1 & 90 & -0.18586 \\
\hline ; 30 & 32 & 34 & 36 & 1 & 90 & 9.44401 \\
\hline
\end{tabular}

; Sidechain improper dihedrals
7
910
8
2
0
$50 ; \mathrm{TYR}$

\#ifdef POSRES

\#include "posre.itp"

\#endif 


\section{Appendix 2: Bonded parameters for sorbitol CG model}

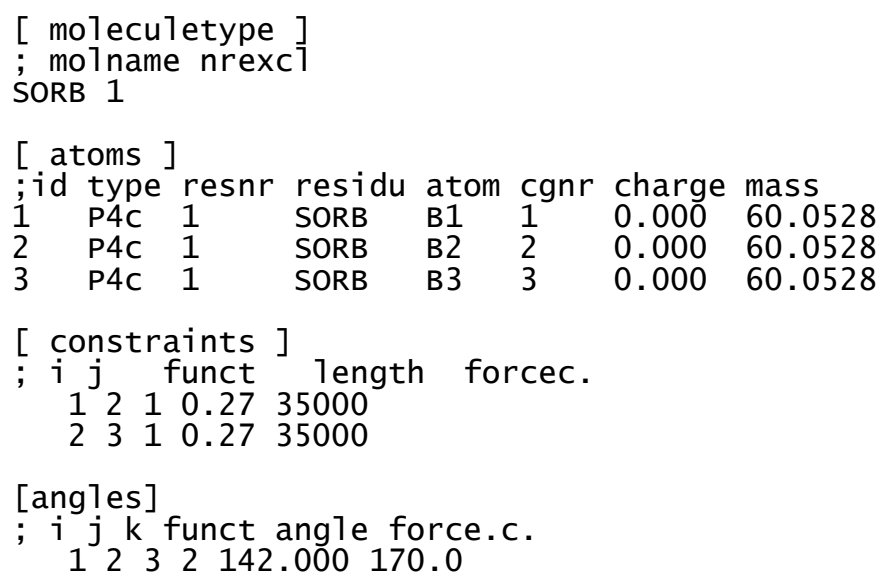




\section{Appendix 3: forcefield parameters for $\mathrm{P4c}$ bead type.}

P4C $72.0 \quad 0.000$ A $0.0 \quad 0.0$

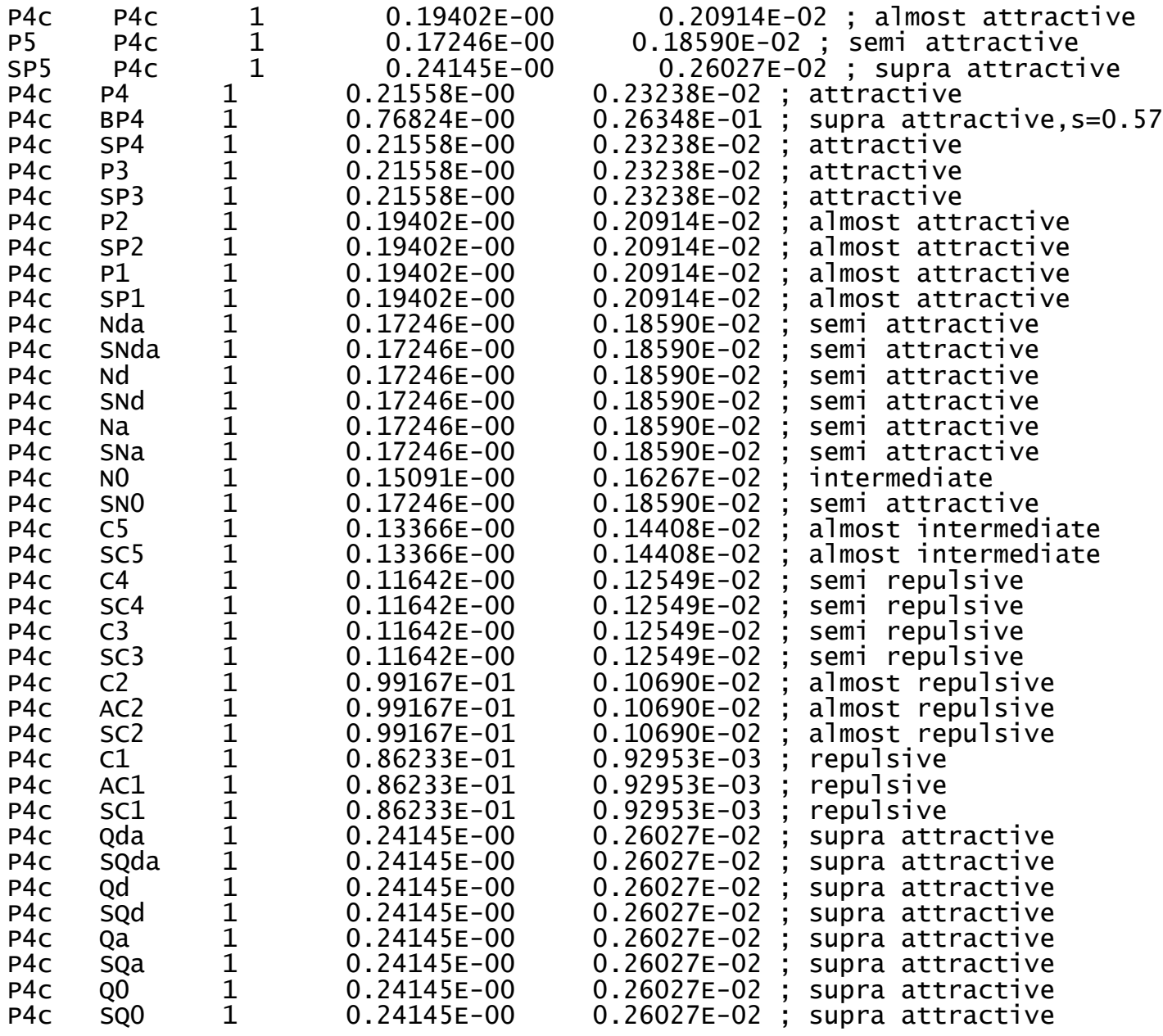

